\title{
Índice de adequação do gerenciamento de resíduos sólidos urbanos como ferramenta para o planejamento: aplicação no estado de São Paulo
}

\author{
Adequacy index of municipal solid waste management \\ as a planning tool: use in the state of São Paulo, Brazil
}

Rodrigo Custódio Urban'

口-

\section{RESUMO}

Considerando as diretrizes de gestão e gerenciamento da Política Nacional dos Resíduos Sólidos e a necessidade de uma visão ampla da problemática dos resíduos sólidos urbanos, este artigo propôs uma metodologia qualitativa de análise regional dos resíduos sólidos urbanos, baseada em um índice aplicado como ferramenta de planejamento. Apresentou-se, também, um panorama dos resíduos sólidos urbanos gerados no estado de São Paulo. Para o desenvolvimento do método, foram usados dados de geração de resíduos, população e desenvolvimento humano. Planilhas de cálculo e sistema de informações geográficas foram empregadas como ferramentas. Um índice de adequação do gerenciamento dos resíduos sólidos urbanos foi gerado a partir da atribuição de pesos para variáveis de população, geração de resíduos sólidos urbanos, coleta seletiva e dos resíduos de serviço de saúde e participação em consórcios intermunicipais. Foi obtida uma equação para estimativa da geração de resíduos sólidos urbanos em função da população do estado de São Paulo, com R²=0,9977. O índice de adequação do gerenciamento dos resíduos sólidos urbanos não apresentou tendência espacial, mas possibilitou identificar municípios com maiores problemas na gestão de resíduos sólidos urbanos. Uma análise dos aterros regionais foi realizada, os quais, em sua maioria, estão localizados próximos aos municípios de maior porte do estado de São Paulo. Existe necessidade de aumentar a quantidade de aterros regionais próximos aos municípios de menor porte da parte Oeste do estado. A metodologia do índice de adequação do gerenciamento dos resíduos sólidos urbanos se mostrou adequada para uma análise regional da problemática de resíduos, podendo ser adaptada para outras áreas de estudo, em regiões administrativas diversificadas. Não foi encontrada correlação entre os dados de geração e de desenvolvimento humano.

Palavras-chave: aterros sanitários; gestão de resíduos sólidos urbanos; saneamento; análise qualitativa regional; aterros regionais; panorama dos resíduos sólidos urbanos no estado de São Paulo.

\begin{abstract}
Given the guidelines for management and administration of the Brazilian Policy for Solid Waste, as well as the need for a broad view of the problematics regarding municipal solid waste, this article proposed a qualitative methodology of regional analysis of municipal solid waste, based on an index used as a planning tool. An overview of the municipal solid waste generated in the state of São Paulo was presented, since it was the area under study. Data for waste generation, population and human development were used for the method development. Measurement spreadsheets and geographic information system were used as tools. An adequacy index of municipal solid waste management (IMSWM) was generated based on weight attribution for variables of population, municipal solid waste generation, selective collection, collection of waste from healthcare services, and participation in municipal consortia. An equation for estimating the generation of municipal solid waste based on the population of the state of São Paulo was obtained, with $\mathrm{R}^{2}=0.9977$. The adequacy index of municipal solid waste management did not show a spatial trend, though it was possible to identify municipalities with greater problems regarding the municipal solid waste management. An analysis of regional landfills was carried out. Most of those from the state of São Paulo are located close to larger municipalities. There is a need of increasing the amount of regional landfills closer to smaller municipalities in the Western part of the state. The methodology of adequacy index of municipal solid waste management seemed adequate for a regional analysis of the waste problematics. It can be adapted to other study areas, in different regions, in several administrative divisions. No correlation between data generation and human development was found.
\end{abstract}

Keywords: sanitary landfill; municipal solid waste management; sanitation; regional qualitative analysis; regional landfill; overview of municipal solid waste in the state of São Paulo. 


\section{INTRODUÇÃO}

Após a Revolução Industrial, o consequente e constante aumento da industrialização contribuiu para a ampliação da geração de resíduos sólidos urbanos (RSU). Apesar de alternativas para a reutilização desses resíduos, como reciclagem, compostagem, geração de energia etc., o baixo percentual daqueles com destinos finais ambientalmente adequados torna inevitável a existência de formas de disposição final, tais como os aterros sanitários.

$\mathrm{O}$ aterro sanitário pode ser considerado uma técnica adequada para a disposição dos RSU no solo, sem causar danos à saúde pública e minimizando os impactos ambientais (CARVALHO \& OLIVEIRA, 2010). Em tal método, são utilizados princípios de engenharia para o confinamento do resíduo sólido na menor área possível e a redução de seu volume ao mínimo permissível, cobrindo-o com uma camada de solo na conclusão de cada jornada de trabalho e com estruturas para adequada drenagem de gases e líquidos. Na base de sua construção, são colocados materiais impermeabilizantes de modo a proteger o solo e a água subterrânea de contaminações provenientes da degradação dos RSU. No final da construção, insere-se uma camada de cobertura para isolamento do aterro e controle da saída de gases e entrada de água (TCHOBANOGLOUS \& O’LEARY, 1994).

Outra realidade brasileira da disposição final de RSU - inadequada por sinal - é o vazadouro a céu aberto, ou lixão. Este é o pior cenário imaginável, com a descarga dos resíduos sem quaisquer medidas de proteção ao ambiente ou à saúde pública (BIDONE \& POVINELLI, 1999).
A situação da disposição de resíduos no Brasil vem melhorando gradualmente, conforme dados compilados de Jucá (2003) e do Instituto Brasileiro de Geografia e Estatística - IBGE (2010), apresentados na Figura 1. Pode-se perceber uma diminuição da quantidade diária de resíduos dispostos inadequadamente a partir de 1989 a 2008, assim como um crescimento dos resíduos lançados em aterros sanitários.

Especificamente no estado de São Paulo, conforme dados da Companhia Ambiental do Estado de São Paulo - CETESB (2013), são geradas cerca de 39 mil toneladas diárias de RSU. As condições da disposição final dos resíduos sólidos nos 645 municípios do estado melhoraram de 1997 a 2013, segundo o Inventário Estadual de Resíduos Sólidos Domiciliares, publicado pela CETESB nesse período. A evolução dos índices de qualidade dos aterros de resíduos (IQR) pode ser observada na Figura 2. Em 2011, houve uma mudança no cálculo do índice, excluindo-se a categoria 'controlado' e classificando os aterros apenas em inadequados ou adequados.

Apesar da evolução apresentada, ainda existem muitos problemas referentes à disposição de resíduos no Brasil, principalmente em municípios de pequeno porte, com restrições financeiras. A situação da disposição diária de RSU por tamanho de municípios pode ser observada na Figura 3. As porcentagens relativas às unidades de compostagem, triagem e incineração são muito baixas e sequer aparecem no gráfico.

Como alternativa às pequenas cidades, que enfrentam o problema da disposição dos RSU, sugere-se a criação de aterros sanitários comuns, utilizados por um consórcio de cidades, que contribuem para

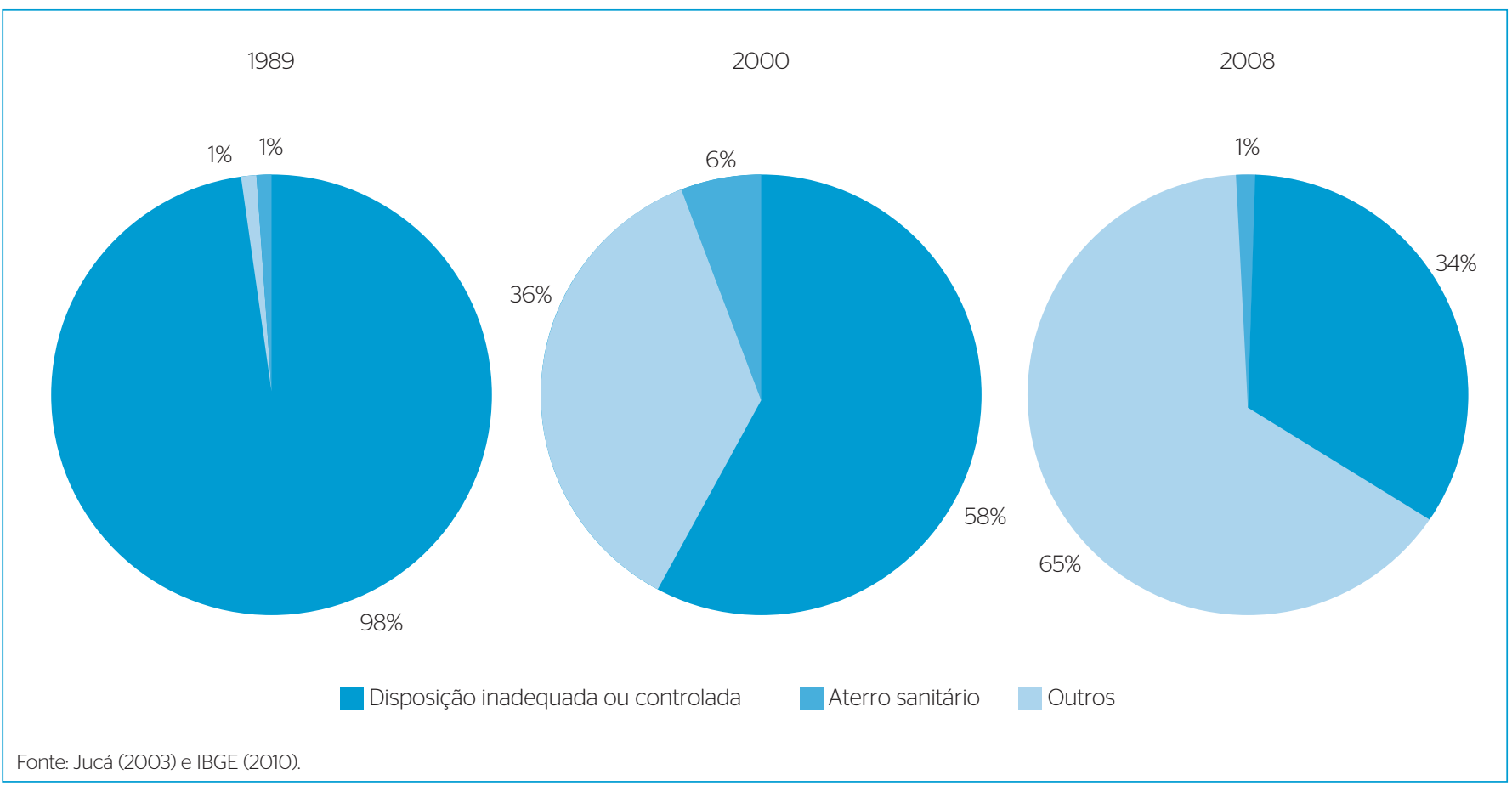

Figura 1 - Disposição final diária de resíduos sólidos urbanos no Brasil. 
a diminuição dos custos do processo. Além disso, existem aterros sanitários particulares que cumprem essa função devido aos locais estratégicos em que são instalados.

O aumento de aproveitamento dos resíduos, por meio de processos como reciclagem e compostagem, além de ampliar a vida útil dos aterros sanitários, auxilia em questões ambientais e segue os preceitos da recente Política Nacional de Resíduos Sólidos - PNRS (BRASIL, 2010).

Considerando as diretrizes da PNRS (BRASIL, 2010) para a gestão integrada, o gerenciamento e o melhor aproveitamento dos resíduos,

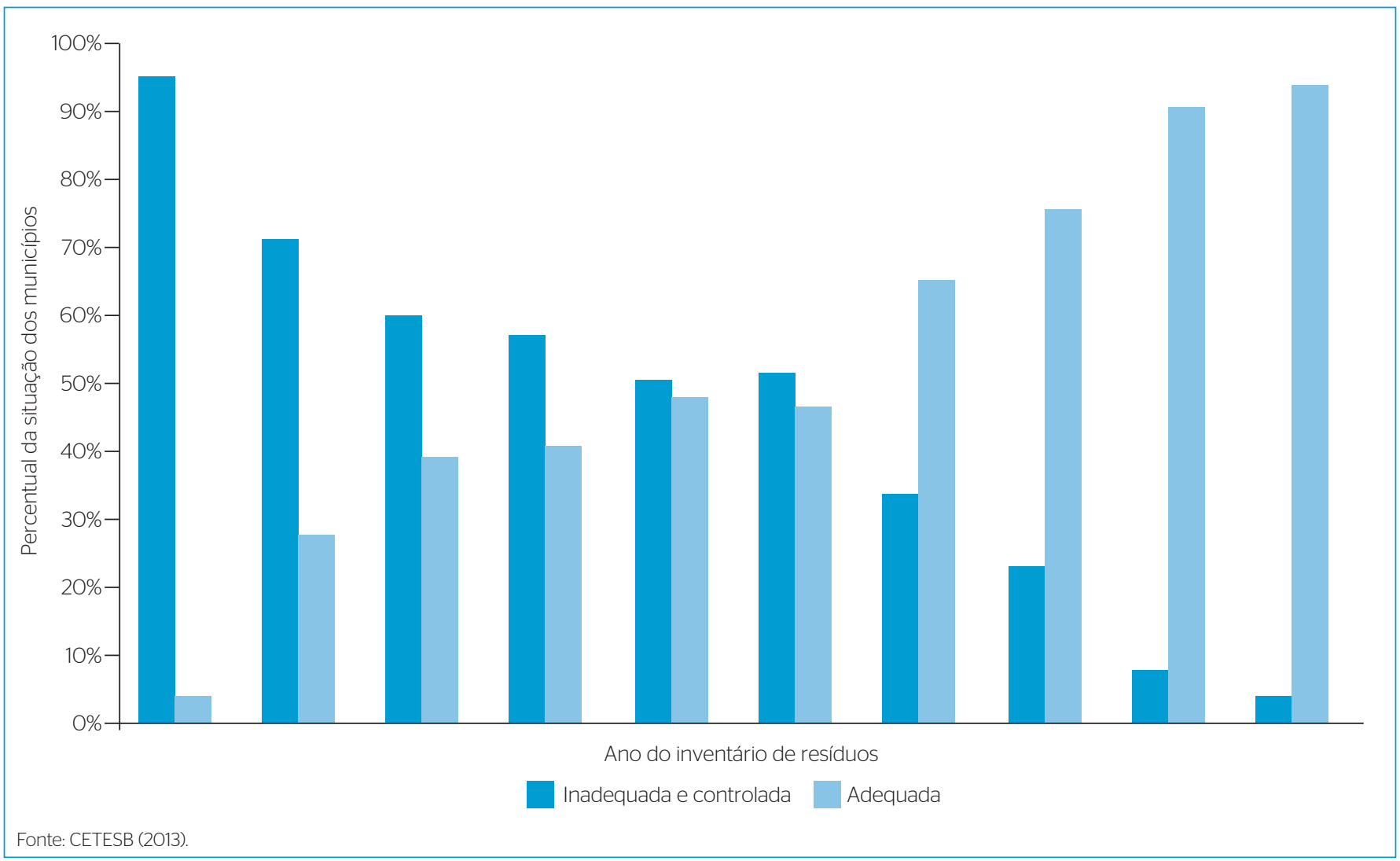

Figura 2 - Situação da disposição final de resíduo domiciliar (\%) dos municípios do estado de São Paulo, de 1997 a 2013.



Figura 3 - Quantidade diária de resíduos sólidos urbanos coletados e/ou recebidos, por unidade de destino, segundo os grupos de tamanho dos municípios, Brasil, 2008. 
em conjunto com a necessidade dos gestores de uma visão mais ampla do conjunto de problemas regionais dos resíduos sólidos, foi proposta, no presente artigo, uma metodologia qualitativa da análise regional dos resíduos sólidos, gerando conjuntamente um panorama da gestão dos RSU no estado de São Paulo.

\section{METODOLOGIA}

Para o presente trabalho, utilizou-se como área de estudo o estado de São Paulo, localizado na região Sudeste do Brasil. Este apresenta uma densidade demográfica de 166,23 hab. $\mathrm{km}^{-2}$, sendo intensamente industrializado, urbanizado e consequentemente com grande geração de resíduos (IBGE, 2014).

A partir da consulta ao banco de dados do Sistema Nacional de Informações sobre Saneamento - SNIS 2012 (BRASIL, 2014), do IBGE (2005) e da Fundação Sistema Estadual de Análise de Dados (SEADE, 2014), foram obtidas informações de geração de resíduos, população, limites geográficos e qualidade de vida municipais.

Os dados foram tabulados e organizados e as variáveis pertinentes foram selecionadas e espacializadas, por meio de planilhas de cálculo e do sistema de informação geográfica (SIG), conforme descrito a seguir.

Para as próximas etapas da metodologia, utilizou-se o programa Microsoft Excel 2007:

- avaliação dos dados tabulados do SNIS 2012;

- análise de regressão linear dos dados de população versus geração de resíduos dos municípios;

- seleção dos critérios adotados;

- definição dos pesos e intervalos numéricos associados a eles;

- somatória dos pesos;

- análise da correlação entre variáveis de produção de resíduos, qualidade de vida e taxa de urbanização dos municípios.

Nas etapas subsequentes, o desenvolvimento do trabalho seguiu em ambiente do SIG, com o programa QGIS 2.4 - Chugiak:

- recorte dos dados do estado de São Paulo a partir da base de dados geográficos (IBGE, 2005);

- inserção dos dados tabulados e calculados no SIG;

- junção das tabelas de atributos das duas fontes primárias (SNIS 2012 e IBGE);

- reclassificação da somatória dos pesos dados a cada município;

- geração do mapa da adequação de gerenciamento dos resíduos;

- geração do mapa de aterros regionais.

A classificação das variáveis depende dos critérios adotados. Os critérios do presente estudo foram definidos por meio da análise dos dados de geração das diferentes formas de resíduos, dentro da limitação das informações fornecidas pelo SNIS 2012 (BRASIL, 2014).
Considerou-se que a problemática gerencial da prévia disposição final dos resíduos sólidos nos municípios, englobando os preceitos da PNRS, depende da população, do total de RSU gerados, do transporte desses resíduos para o destino final (no caso, os aterros sanitários), da prática de ações de coleta seletiva, da participação do município em algum consórcio intermunicipal e da coleta diferenciada dos resíduos de serviços de saúde (RSS).

Enfatiza-se que não foi utilizado o critério das condições das instalações da disposição final de resíduos, divulgado anualmente pela CETESB, por meio do IQR, pois pretendeu-se verificar o gerenciamento municipal dos resíduos antes de sua disposição final.

Os critérios foram divididos em três categorias, cada uma com um peso diferente: 0,1 e 2 . O valor “0” considera que tal critério é bem atendido pelo município, não contribuindo para os problemas mais urgentes; "1" expressa que tal local se encontra na média dos municípios semelhantes, gerando uma preocupação em relação às condições futuras desse critério. O valor "2" indica uma situação mais urgente para o município, naquele critério, em que medidas devem ser adotadas o quanto antes para uma melhor gestão dos RSU.

Para a separação em relação às faixas populacionais, empregou-se como referência o trabalho de Campos (2012), considerando a média de resíduos gerados per capita no Brasil. Quanto a essa média, aplicou-se um intervalo de $\pm 0,5 \mathrm{~kg}$.hab ${ }^{-1}$.dia ${ }^{-1}$ para situar os municípios que estão acima ou abaixo da média geral.

A exportação de resíduos também foi relativa em relação ao tamanho do município. Por exportação, entende-se que o município envia os RSU para outro. Para uniformizar esse critério em cidades de pequeno porte, utilizou-se a indicação da CETESB (2005) para aterros em valas como forma de minimizar o problema dos RSU. Em função da discussão que essa medida gera, considerou-se que tanto o envio dos resíduos para outro município, quanto o uso do aterro em valas, não contribuem significativamente para o aumento de problemas gerenciais nesses municípios. Em cidades maiores, os gastos com o transporte de resíduos são mais problemáticos. Os critérios estão apresentados na Tabela 1.

A análise qualitativa consistiu na adoção de pesos (entre 0 e 2) para intervalos relacionados aos critérios da Tabela 1. Após essa etapa, foram somados os valores de cada um para os municípios dentro da área de estudo (no caso, o estado de São Paulo). O valor mínimo possível seria 1 e o máximo, 9. Dentro desse intervalo, os municípios foram reclassificados em relação ao nível de adequação do gerenciamento de resíduos, o que gerou a classificação apresentada na Tabela 2.

Para um município ter adequação de gerenciamento alta, considerou-se que ele poderia ter apenas um ponto a mais que o mínimo possível. Para tal localização ser de adequação de gerenciamento muito baixa, deveria ter pontuação máxima em todos os critérios. O intervalo restante (de 3 a 8 ) foi dividido igualmente entre as duas categorias intermediárias. 


\section{RESULTADOS E DISCUSSÃO}

Primeiramente, serão apresentados os resultados gerais do estado de São Paulo. Além de indicarem um panorama, esses dados foram usados como subsídio para a definição dos pesos e uma posterior análise qualitativa. Na Tabela 3, os dados totais e relativos de alguns aspectos do SNIS 2012 (BRASIL, 2014) são mostrados.

Uma primeira observação a ser feita é sobre a fonte dos dados. A pesquisa SNIS é respondida pelos próprios municípios e/ou empresas de saneamento. Com um aspecto mais abrangente, tem detalhes de custos com mão de obra, resíduos gerados, esgoto e água tratados, entre outros. Entretanto, qualquer desconformidade quanto à realidade pode ser causada por erros nas informações passadas ao Ministério das Cidades (BRASIL, 2014).

Em relação à Tabela 3, pode-se notar que cerca de $77 \%$ do total de municípios de São Paulo respondeu à pesquisa. Portanto, os dados serão trabalhados baseando-se nesse percentual, considerando apenas os municípios com informações fornecidas. Apesar de somente cerca de $31 \%$ deles afirmarem que exportam resíduos, a população total e os resíduos gerados nesses municípios representam, respectivamente, aproximadamente de 65 a $68 \%$ do total. Isso é bastante influenciado pela cidade de São Paulo, que tem mais de 11 milhões de pessoas atendidas pelo sistema de coleta.

Quase 60\% dos municípios declaram possuir um programa de coleta seletiva, mas apenas $52 \%$ da população é atendida pelo serviço, mesmo considerando cidades grandes como São Paulo e Campinas. Isso se deve ao fato deles não atenderem, necessariamente, toda a população do município. Os consórcios ainda são incipientes, até mesmo pela proximidade da publicação da legislação (BRASIL, 2010) com a pesquisa (2012), representando em torno de 10 a 12\% do número de municípios, população e resíduos gerados.

Os RSS são declaradamente coletados de forma diferenciada por mais de $95 \%$ dos municípios, atendendo a mais de $98 \%$ da população, o que é um aspecto positivo.

Em relação à geração de resíduos per capita, percebe-se que os municípios que exportam resíduos geram cerca de $50 \mathrm{~g}$ a mais por habitante do que o valor total do Estado. Já aqueles integrantes de consórcio geram aproximadamente $60 \mathrm{~g}$ a menos por habitante. Esses valores são relativamente próximos e demonstram certa uniformidade na geração média de resíduos do Estado.

De modo a entender melhor a relação entre população e geração de resíduos no estado de São Paulo, conduziu-se uma regressão linear com os dados estudados. Na Figura 4, pode-se observar o seu resultado, com a equação da reta, o valor do coeficiente de determinação e a dispersão dos pontos. Deve-se enfatizar que o dado referente à cidade de São Paulo foi retirado da visualização, por ter um valor bastante superior aos demais municípios, dificultando a interpretação.

O coeficiente de determinação indica que $99,77 \%$ da massa de resíduos são explicados pelo modelo. Este é um ajuste bom, que permite

Tabela 2 - Classificação dos municípios em relação à soma de pesos atribuídos.

\begin{tabular}{l|c}
\hline Adequação de gerenciamento & Soma de pesos \\
\hline Alta & $1-2$ \\
\hline Média & $3-5$ \\
\hline Baixa & $6-8$ \\
\hline Muito baixa & 9 \\
\hline
\end{tabular}

Tabela 1 - Critérios adotados na avaliação qualitativa e intervalos das categorias.

\begin{tabular}{|c|c|c|c|c|c|}
\hline \multirow{3}{*}{ Critério } & \multirow{2}{*}{ Faixa populacional municipal } & \multirow{2}{*}{ Média brasileira } & \multicolumn{3}{|c|}{ Intervalo da categoria } \\
\hline & & & 0 & 1 & 2 \\
\hline & (número de habitantes) & & \multicolumn{3}{|c|}{$\left(\mathrm{kg} \cdot \mathrm{hab}^{-1} \cdot \mathrm{dia}^{-1}\right)$} \\
\hline \multirow{6}{*}{ Resíduos gerados per capita } & Até 30 mil & 0,81 & $(0-0,31)$ & $(0,31-1,31)$ & $(1,31-3)$ \\
\hline & 30 a 100 mil & 0,77 & $(0-0,27)$ & $(0,2-1,27)$ & $(1,27-3)$ \\
\hline & 100 a 250 mil & 0,81 & $(0-0,31)$ & $(0,31-1,31)$ & $(1,31-3)$ \\
\hline & 250 mil a 1 milhão & 0,97 & $(0-0,47)$ & $(0,47-1,47)$ & $(1,47-3)$ \\
\hline & 1 a 3 milhões & 1,19 & $(0-0,69)$ & $(0,69-1,69)$ & $(1,69-3)$ \\
\hline & Acima de 3 milhões & 0,95 & $(0-0,45)$ & $(0,45-1,45)$ & $(1,45-3)$ \\
\hline \multirow{2}{*}{ Exportação dos resíduos } & Até 25 mil & - & - & Não/Sim & - \\
\hline & Acima de 25 mil & - & - & Não & Sim \\
\hline \multirow{2}{*}{ PCS } & - & \multicolumn{4}{|c|}{ População atendida } \\
\hline & - & $12 \%$ & $(\mathrm{O} \%)$ & (O a 50\%) & $(>50 \%)$ \\
\hline Coleta RSS & - & - & Sim & - & Não \\
\hline Consórcio & - & - & $\operatorname{Sim}$ & Não & - \\
\hline
\end{tabular}

PCS: Programa de Coleta Seletiva; Coleta RSS: Existe coleta diferenciada dos resíduos de serviços de saúde?; Consórcio: O município faz parte de algum consórcio intermunicipal? Fonte da média brasileira de geração de RSU: Campos (2012). 
considerar a equação da reta proposta como um modelo estimativo da quantidade anual de RSU gerada nos municípios paulistas. Essa equação pode servir como uma estimativa preliminar quando não se dispõe de outros dados, ou quando se propõe um estudo baseado no crescimento populacional. Há de se precaver quanto às diferenças de consumo relacionadas com o poder aquisitivo, existindo diferenças de local para local e em relação ao tempo (CAMPOS, 2012). Entretanto, regionalmente, o modelo (Equação 1) se apresenta viável.

MRG=0,3532.Pop. $-1553,4$

onde:

$\mathrm{MRG}=$ massa de resíduos gerados $\left(\mathrm{t} \cdot \mathrm{ano}^{-1}\right)$;

Pop = população do local de estudo (habitantes).

\section{Análise da adequação do}

\section{gerenciamento dos resíduos sólidos urbanos}

Com os critérios e pesos adotados, foi possível obter um mapa que apresenta o índice de adequação do gerenciamento de RSU (IAGR) dos municípios paulistas (Figura 5).

Os municípios considerados "sem informação" foram aqueles que não incluíram os dados para o SNIS 2012 (BRASIL, 2014). De restante, é possível observar a distribuição espacial dos municípios com a adequação de gerenciamento geral considerada baixa, média e alta. Nenhum município paulista atingiu a somatória de 9 para se enquadrar na categoria da adequação de gerenciamento muito baixa.

Ainda segundo o mapa da Figura 5, nota-se que poucos municípios se enquadram na categoria de adequação de gerenciamento alta. O critério para esta foi mais restritivo, pois considera-se que os RSU são um problema municipal considerável. A maior parte dos municípios analisados encontra-se dentro da categoria média, tendo um ou outro aspecto que merece mais atenção, quando analisados individualmente. Mais municípios se adequam na categoria baixa do que na alta. Apesar da faixa de valores dos grupos ser diferente, há mais municípios em estado de maior atenção do que aqueles onde a gestão dos resíduos atende à maioria dos critérios considerados.

Não foi possível verificar nenhuma tendência espacial da distribuição das categorias a partir dos dados obtidos.

Algumas limitações podem ser observadas no método qualitativo. Considerando os valores de população e geração de resíduos a partir da geração per capita, problemas como o da cidade de São Paulo (grande geração de resíduos) acabam subestimados. Entretanto, como a ideia é uma análise regional, para unidades de gestão como os consórcios, esse problema acaba não tendo maiores consequências. Em grandes cidades, pode-se pensar em adaptar o método para

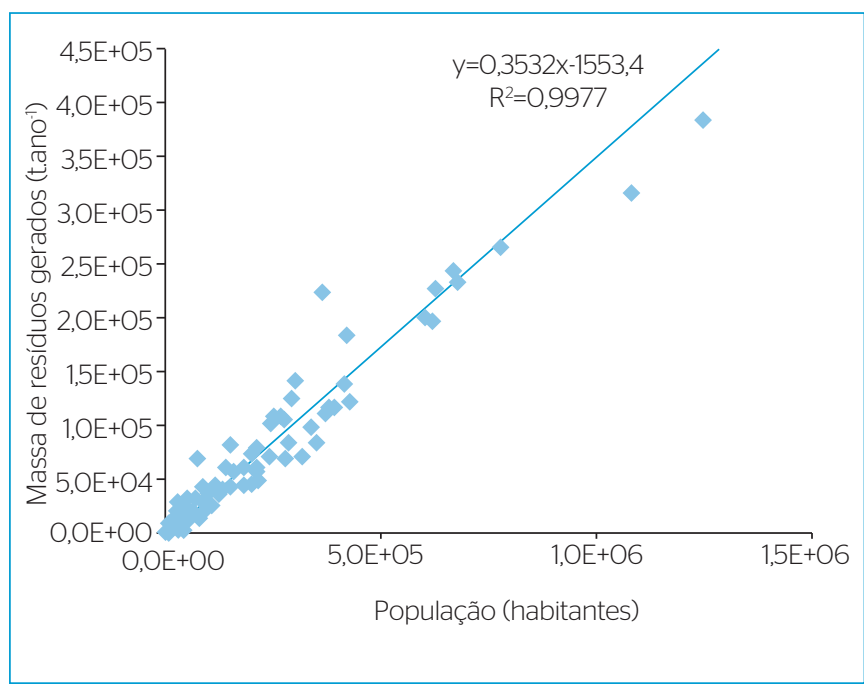

Figura 4 - Regressão linear dos dados de população versus resíduos gerados no estado de São Paulo.

Tabela 3 - Dados totais e relativos sobre a geração de resíduos no estado de São Paulo.

\begin{tabular}{|c|c|c|c|c|}
\hline Dados & $\begin{array}{c}\text { Número } \\
\text { de municípios }\end{array}$ & $\begin{array}{l}\text { População total } \\
\text { (habitantes) }\end{array}$ & $\begin{array}{l}\text { Resíduos gerados } \\
\text { (t.ano-1) }\end{array}$ & $\begin{array}{l}\text { Resíduos per capita } \\
\left(\mathrm{kg} \cdot \mathrm{hab}^{-1} \cdot \mathrm{dia}^{-1}\right)\end{array}$ \\
\hline Municípios com dados informados & 497 & 37.760 .989 & $12.565 .109,0$ & 0,912 \\
\hline \% em relação ao total de municípios de São Paulo & $77,05 \%$ & $86 \%$ & - & - \\
\hline Municípios que exportam resíduos sólidos urbanos & 154 & 24.386 .618 & $8.548 .768,5$ & 0,960 \\
\hline \% em relação aos municípios com dados & $30,99 \%$ & $64,58 \%$ & $68,04 \%$ & - \\
\hline Municípios com coleta seletiva & 301 & 19.468 .176 & $342.116,8$ & 0,048 \\
\hline \% em relação aos municípios com dados & $60,56 \%$ & $51,56 \%$ & $2,72 \%$ & - \\
\hline Municípios integrantes de consórcio & 62 & 3.932 .999 & $1.224 .256,3$ & 0,853 \\
\hline \% em relação aos municípios com dados & $12,47 \%$ & $10,42 \%$ & $9,74 \%$ & - \\
\hline Municípios com coleta diferenciada de resíduos sólidos de saúde & 475 & 37.209 .166 & $64.281,1$ & 0,005 \\
\hline \% em relação aos municípios com dados & $95,57 \%$ & $98,54 \%$ & $0,51 \%$ & \\
\hline
\end{tabular}


uma análise de distritos, bairros ou outras unidades de gestão, melhorando essa visão local da adequação do gerenciamento de resíduos.

\section{Análise de aterros regionais}

A fim de melhor observar as características regionais da gestão de RSU, elaborou-se outro mapa, englobando as cidades com aterros que recebem os resíduos de mais de um município. Foram excluídos os aterros que recebem resíduos de dois municípios (um além do município de origem), pois estes não foram considerados como regionais. Dentro da soma dos totais de resíduos gerados e da população, foram considerados também os valores dos municípios de origem dos aterros (quando os dados estavam disponíveis). Diante disso, os totais de população e geração dos resíduos são ligeiramente diferentes daqueles apresentados na Tabela 3.

$\mathrm{Na}$ Figura 6, são apresentados os municípios que exportam seus resíduos para os aterros regionais mencionados na legenda.

Tal análise permite uma visão regional e indica algumas características espaciais relevantes. Os municípios que exportam os resíduos estão, em sua maioria, concentrados nas porções Nordeste e Sudeste do estado, com destaque maior para a segunda. Neste local, estão inseridas as regiões metropolitanas de São Paulo, Campinas, Santos e Sorocaba (DEDECCA et al., 2009), abrangendo boa parte da população do estado e consequentemente da geração de resíduos.

Na região NE (abrangendo um pouco da $\mathrm{N}$ ), encontram-se importantes polos do estado de São Paulo, como os municípios de São José do Rio Preto e Ribeirão Preto.

Apesar da proximidade com as maiores cidades do estado, os aterros regionais estão, em sua maioria, localizados em cidades menores próximas a elas. Isso torna, portanto, evidente que estes aterros, muitas vezes particulares, foram projetados para receber os resíduos dessas grandes cidades. Com isso, municípios menores das proximidades se beneficiam, tendo uma opção mais viável do que a construção de um aterro próprio. Na Tabela 4, observa-se o município sede dos aterros considerados regionais, o número de municípios declaradamente atendidos pelo serviço, a população atendida e a massa de resíduos per capita.

Ainda, na Tabela 4, nota-se a existência de 26 aterros regionais. O de Paulínia atende ao maior número de municípios (24) e o de Caieiras, à maior população e, consequentemente, recebe a maior quantidade de

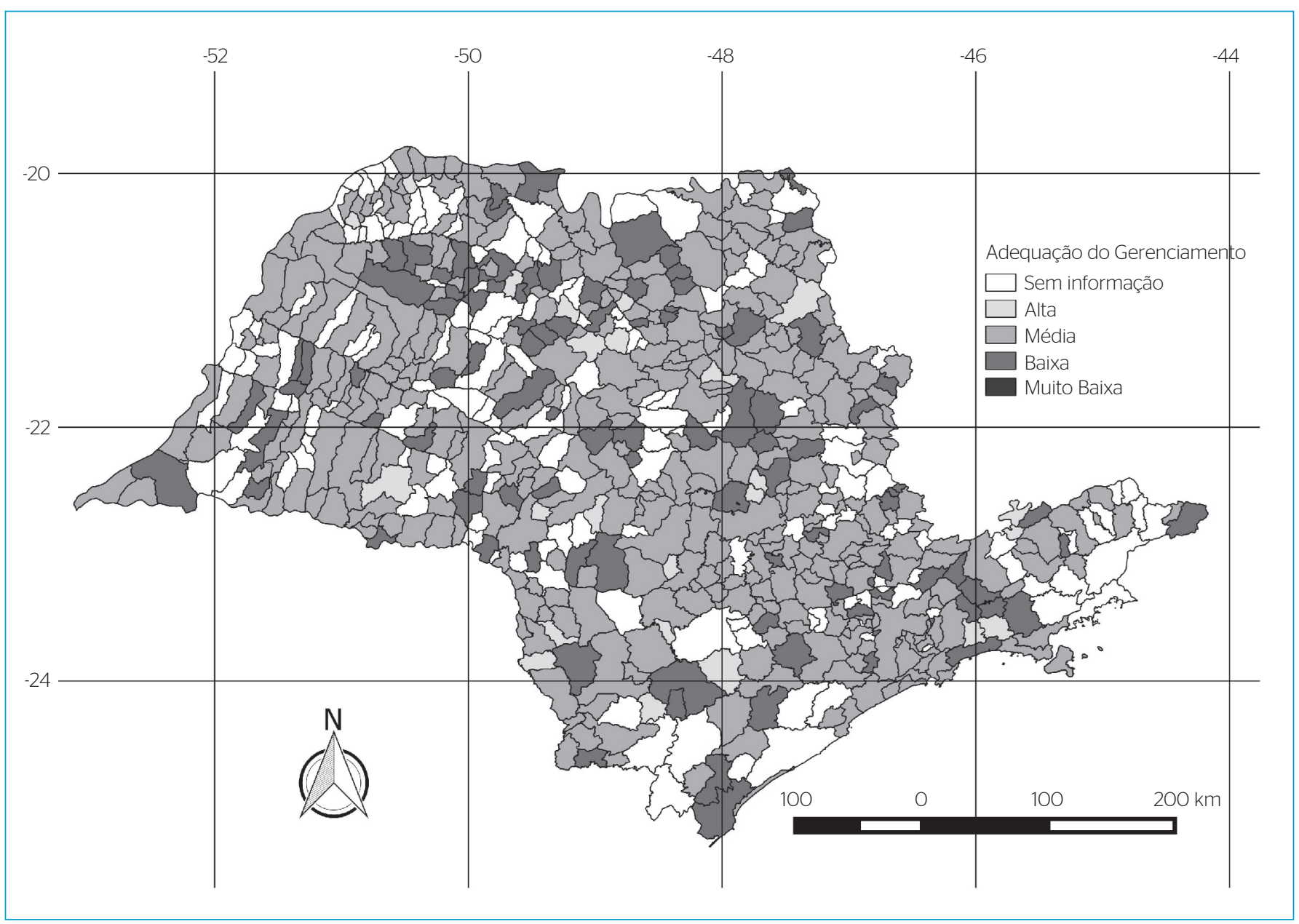

Figura 5 - Mapa do índice de adequação do gerenciamento de resíduos sólidos urbanos dos municípios paulistas. 
resíduos anuais. Esses dois aterros encontram-se nas regiões metropolitanas de Campinas e São Paulo, respectivamente.

Além disso, os valores em negrito na Tabela 4 representam os aterros regionais que têm a geração per capita diária de resíduos acima da média do estado de São Paulo. Com exceção do aterro de Meridiano, todos têm em comum a proximidade com alguma cidade grande do estado.

Também é possível destacar os aterros de Jambeiro, Santa Isabel e Santos, com os maiores valores de resíduos per capita. Os últimos três citados recebem resíduos de municípios litorâneos, com atividades turísticas bem desenvolvidas. Os turistas tornam a população desses municípios flutuante, principalmente no verão, gerando uma quantidade grande de resíduos. Esse aspecto evidencia outro problema, pois tais cidades estão parcialmente inseridas em áreas de proteção ambiental, diminuindo a área disponível para a construção de aterros. Além disso, o transporte dessa massa é custoso em função da barreira da Serra do Mar, que divide o litoral paulista do restante do estado.

Os altos valores de resíduos per capita e o atendimento dos aterros regionais a municípios de grande porte evidenciam que ainda há bastante espaço para a gestão consorciada dos resíduos, principalmente pelos municípios de menor porte localizados na porção Oeste do estado de São Paulo. A necessidade de aterros regionais é maior em função das recentes exigências da PNRS (BRASIL, 2010).

\section{Correlação dos dados da geração de resíduos com os de desenvolvimento humano}

Para tentar identificar uma relação entre a quantidade de RSU gerados no estado de São Paulo e o desenvolvimento humano dos municípios paulistas, foram usados dados do SEADE (2014). Esses representam a porcentagem de urbanização do município e o valor do índice de desenvolvimento humano municipal (IDHM), que é uma adaptação brasileira do valor do IDH global, pensando no aspecto municipal do desenvolvimento. Os componentes de montagem do índice são os mesmos do global, adaptados à realidade municipal: longevidade, educação e renda (IPEA, 2013). Na Tabela 5, podem ser notadas as correlações entre as variáveis consideradas.

Apesar da hipótese inicial de uma correlação entre a quantidade de resíduos gerados e o IDHM, isso não foi identificado para as variáveis do estado de São Paulo. No entanto, sabe-se que o aumento de

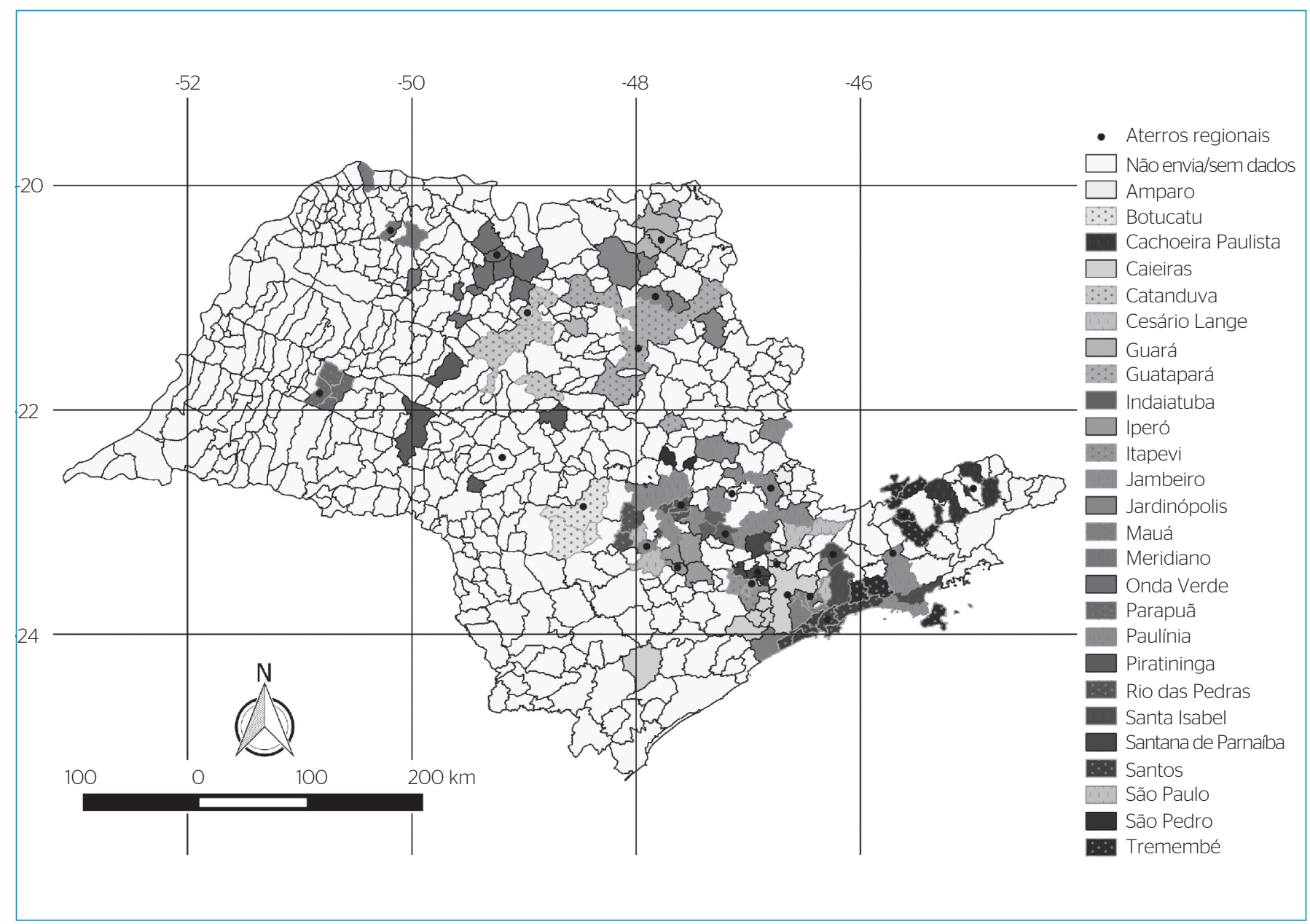

Figura 6 - Municípios que exportam resíduos sólidos urbanos para aterros regionais (listados na legenda) no estado de São Paulo. 
renda e o consequente incremento na qualidade de vida influenciam na geração de resíduos, como apontado por Campos (2012) e Liu e Wu (2010).
Outra possível justificativa para o resultado não satisfatório obtido consiste no fato de o incremento da renda influenciar mais na composição gravimétrica do resíduo do que na massa propriamente dita.

Tabela 4 - Dados dos aterros regionais do estado de São Paulo.

\begin{tabular}{|c|c|c|c|c|}
\hline Municipio/Aterro regional & Total de municípios & $\begin{array}{l}\text { População atendida } \\
\text { (habitantes) }\end{array}$ & Resíduos (t.ano-1) & $\begin{array}{l}\text { Resíduos per capita } \\
\left(\mathrm{kg} \cdot \mathrm{hab}^{-1} \cdot \mathrm{dia}^{-1}\right)\end{array}$ \\
\hline Amparo/SP & 4 & 37.013 & $10.395,2$ & 0,77 \\
\hline Botucatu/SP & 2 & 153.712 & $41.640,4$ & 0,74 \\
\hline Cachoeira Paulista/SP & 5 & 209.720 & $44.442,8$ & 0,58 \\
\hline Caieiras/SP & 8 & 11.964 .590 & $4.264 .258,4$ & 0,98 \\
\hline Guará/SP & 5 & 165.986 & $38.442,4$ & 0,63 \\
\hline Guatapará/SP & 13 & 883.249 & $264.216,9$ & 0,82 \\
\hline Indaiatuba/SP & 2 & 275.355 & $78.499,4$ & 0,78 \\
\hline Iperó/SP & 3 & 674.890 & $219.657,6$ & 0,89 \\
\hline Jardinópolis/SP & 4 & 106.461 & $28.862,5$ & 0,74 \\
\hline Mauá/SP & 6 & 2.617 .441 & $900.374,3$ & 0,94 \\
\hline Meridiano/SP & 3 & 104.251 & $38.772,8$ & 1,02 \\
\hline Onda Verde/SP & 8 & 540.793 & 188.007,1 & 0,95 \\
\hline Parapuã/SP & 3 & 43.354 & $10.811,1$ & 0,68 \\
\hline Paulínia/SP & 24 & 2.126 .935 & $639.360,3$ & 0,82 \\
\hline Piratininga/SP & 4 & 317.656 & $122.349,4$ & 1,06 \\
\hline Rio das Pedras/SP & 5 & 65.961 & $20.716,2$ & 0,86 \\
\hline Santa Isabel/SP & 2 & 516.781 & $273.114,8$ & 1,45 \\
\hline Total (\%) & 145 (29,18\%) & $25.174 .541(66,67 \%)$ & $8.815 .456,5$ (70,16\%) & \\
\hline
\end{tabular}

Os valores em negrito representam os valores de resíduos per capita acima da média do Estado de São Paulo.

Tabela 5 - Correlação entre variáveis da geração de resíduos e dos aspectos de qualidade de vida municipal.

\begin{tabular}{|c|c|c|c|c|c|}
\hline Variável & $\begin{array}{l}\text { Total de resíduos } \\
\text { sólidos urbanos }\end{array}$ & População total & Resíduos per capita & \% Urbanismo & $\begin{array}{l}\text { Índice de desenvolvimen- } \\
\text { to humano municipal }\end{array}$ \\
\hline RSU & 1 & - & - & - & - \\
\hline População & 0,998853 & 1 & & & \\
\hline Resíduos per capita & 0,058636 & 0,040145 & 1 & - & - \\
\hline \% Urbanismo & 0,099824 & 0,103988 & 0,183327 & 1 & - \\
\hline
\end{tabular}

RSU: resíduos sólidos urbanos; \% Urbanismo: grau de urbanismo; IDHM: Indice de desenvolvimento humano municipal. Em negrito, o valor de correlação significativa. 
Resíduos de áreas com população de maior renda têm quantidades superiores de plásticos e locais de menor renda têm maior quantidade de matéria orgânica, por exemplo. Isso pode ser corroborado pelos estudos de Mancini et al. (2007) e Lawal (2014).

\section{CONCLUSÕES}

A partir dos resultados obtidos, podem ser efetuadas algumas conclusões sobre a análise qualitativa como ferramenta para a gestão dos RSU, sobre o panorama dos RSU no estado de São Paulo sob a ótica municipal e da correlação com as variáveis da qualidade de vida.

Primeiramente, cabe-se ressaltar que a análise qualitativa proposta engloba conjuntamente: o estudo global dos dados da geração de resíduos; o IAGR proposto e a análise de aterros regionais. Sobre esses aspectos, algumas observações podem ser realizadas:

- a análise dos dados da área de estudo é essencial para o entendimento de características intrínsecas à região;

- a regressão linear entre população e massa de resíduos gerados pode ser usada em outras áreas de estudo, buscando identificar equações para estimativas da geração de resíduos;

- o IAGR proposto utilizou variáveis abrangentes que poderiam ser replicadas em outras áreas de estudo: população e geração de resíduos municipais, exportação de RSU, existência de coleta seletiva, coleta diferenciada de RSS e participação em consórcios intermunicipais;

- o IAGR se mostrou satisfatório para identificar áreas prioritárias, regionalmente, em ações de gestão dos resíduos. Essa metodologia pode ser utilizada em outras áreas administrativas como bacias hidrográficas, sub-regiões estaduais ou distritais (em municípios de maior porte);

- o IAGR não é adequado para uma análise específica dos problemas de cada um dos municípios estudados. Esse estudo deve ser feito localmente;
- a análise de aterros regionais permite uma melhor visão espacial de áreas com maior produção de resíduos, deslocamento necessário para a exportação de resíduos e para auxiliar na definição do panorama da área de estudo.

Especificamente sobre o panorama da situação dos RSU no estado de São Paulo, foi possível identificar alguns aspectos:

- a equação da geração de resíduos em função da população pode ser utilizada como estimativa primária desse dado;

- há espaço para o crescimento da coleta seletiva, formação de consórcios e de aterros regionais para os municípios de menor porte no estado de São Paulo;

- os municípios que se enquadram na categoria "adequação de gerenciamento baixa" precisam revisar a sua gestão de resíduos para uma melhor adequação;

- as regiões metropolitanas do estado contam com uma grande quantidade de aterros regionais, com vida útil limitada, podendo gerar um problema para a disposição dos RSU em médio prazo;

- não foi possível identificar uma correlação entre a geração de RSU e os aspectos de qualidade de vida no estado de São Paulo, o que não indica que um não interfira no outro, tornando necessário outro tipo de estudo para essa determinação.

Como sugestão para estudos futuros:

- atualização dos dados conforme novas pesquisas sejam divulgadas;

- estabelecimento das variáveis dos municípios onde a informação não estava disponível;

- identificação de padrões entre a geração de resíduos e os aspectos econômicos e de qualidade de vida;

- comparação e verificação da correlação entre o IAGR e o IQR.

\section{REFERÊNCIAS}

BIDONE, F.R.A. \& POVINELLI, J. (1999) Conceitos básicos de resíduos sólidos. São Carlos: EESC/USP, Projeto Reenge. 120 p.

BRASIL.(2010) Presidência da República. Casa Civil. Leino 12.305, de 02/08/2010. Institui a Política Nacional de Resíduos Sólidos; altera a Lei no 9.605, de 12 de fevereiro de 1998; e dá outras providências. Disponível em: <http://www.planalto. gov.br/ccivil_O3/_ato2007-2010/2010/lei/12305.htm>. Acesso em: 24 jul. 2012.

BRASIL. (2014) Ministério das Cidades. Secretaria Nacional de Saneamento Ambiental. Sistema Nacional de Informações sobre Saneamento: diagnóstico do manejo dos resíduos sólidos urbanos -2012. Brasilia: SNSA/MCIDADES. 143 p.

CAMPOS, H.K.T. (2012) Renda e evolução da geração per capita de resíduos sólidos no Brasil. Engenharia Sanitária e Ambiental, v. 17, n. 2, p. 171-180.
CARVALHO, A.R. \& OLIVEIRA, M.V.C. (2010) Princípios básicos do saneamento do meio. São Paulo: SENAC. 401 p.

COMPANHIA DE TECNOLOGIA DE SANEAMENTO AMBIENTAL CETESB. (2005) Procedimento para implantação de aterro sanitário em valas. São Paulo: CETESB. 34 p.

COMPANHIA DE TECNOLOGIA DE SANEAMENTO AMBIENTAL CETESB.. (2O13) Inventário estadual de resíduos sólidos domiciliares 2013. São Paulo: CETESB. 118 p.

DEDECCA, C.; MONTALI, L.; BAENINGER, R. (2009) Estudo consolidado para o Estado de São Paulo: regiões metropolitanas e pólos regionais. Campinas: FINEP/NEPP/IE. 280 p. 
FUNDAÇÃO SISTEMA ESTADUAL DE ANÁLISE DE DADOS - SEADE. (2014) Portal de estatísticas do Estado de São Paulo: Informações dos municípios paulistas - IMP. Disponível em: <http://produtos.seade.gov. br/produtos/imp/>. Acesso em: 11 jul. 2014.

INSTITUTO BRASILEIRO DE GEOGRAFIA E ESTATISTICAS - IBGE. (2005) Malha municipal digital 2005. Brasília: IBGE.

INSTITUTO BRASILEIRO DE GEOGRAFIA E ESTATISTICAS - IBGE.. (2010) Pesquisa Nacional de Saneamento Básico 2008. Rio de Janeiro: IBGE. 219 p.

INSTITUTO BRASILEIRO DE GEOGRAFIA E ESTATISTICAS - IBGE.. (2014) Estados@: São Paulo. Disponível em: <http://www.ibge.gov.br/ estadosat/perfil.php?sigla=sp>. Acesso em: 20 ago. 2014.

INSTITUTO DE PESQUISA ECONÔMICA APLICADA - IPEA. (2013) Fundação João Pinheiro. Programa das Nações Unidas para o Desenvolvimento. Atlas do Desenvolvimento Humano no Brasil 2013: o IDHM. Disponível em <http://www.atlasbrasil.org.br/2013/pt/o_atlas/ idhm/>. Acesso em: 28 jul. 2014.
JUCÁ, J.F.T. (2003) Disposição final dos resíduos sólidos urbanos no brasil. In: V Congresso Brasileiro de Geotecnia Ambiental, Anais REGEO 2003. Porto Alegre: ABMS

LAWAL, O. (2014) Wasteful disposition: analysis of municipal household waste using geodemographic classification. Waste Management \& Research, v. 32, n. 2, p. 170-174.

LIU, C. \& WU, X.W. (2010) Factors influencing municipal solid waste generation in China: a multiple statistical analysis study. Waste Management \& Research, v. 29, n. 4, p. 371-378.

MANCINI, S.D.; NOGUEIRA, A.R.; KAGOHARA, D.A.; SCHWARTZMAN, J.A.S.; MATTOS, T. (2007) Recycling potential of urban solid waste destined for sanitary landfills: the case of Indaiatuba, SP, Brazil. Waste Management \& Research, v. 25, n. 6, p. 517-523.

TCHOBANOGLOUS, G.; O'LEARY, P.R. (1994) Landfilling. In: KREITH, F. (ed.) Handbook of solid waste management. New York: McGraw-Hill, Inc. 